\title{
Vorgehensmodelle für die rollenbasierte Autorisierung in heterogenen Systemlandschaften
}

Die Autoren
Felix Wortmann
Robert Winter
Dr. Felix Wortmann
Prof. Dr. Robert Winter
Universität St. Gallen
Institut für Wirtschaftsinformatik
Müller-Friedberg-Str. 8
9000 St. Gallen, Schweiz
Robert.Winter@unisg.ch
Eingereicht am 2007-05-08,
nach einer Überarbeitung
angenommen am 2007-09-09
durch Prof. Dr. Buxmann.

\section{Einleitung}

Eine fundamentale Voraussetzung für die Gewährleistung der Sicherheit von Informationssystemen ist die adäquate Verwaltung und Kontrolle von Zugriffsberechtigungen [RuWo06, 127]. Die hiermit verbundenen Aktivitäten, die unter dem Begriff der „Autorisierung“ [JoDi94, $27 \mathrm{f}$; Pern95, 165f.; SaCa02, 137f.] zusammengefasst werden, stellen die Verantwortlichen vor zahlreiche Herausforderungen. Ein unzureichendes Management der Informationssystemarchitekturen führte in der Vergangenheit insbesondere bei mittleren und großen Unternehmen zu Redundanzen oder Lücken in der Systemlandschaft [Wint03, 318f.]. Im Umfeld der
Autorisierung besitzen die einzelnen Systeme daher meist eigenständige Funktionen und Module, die in der Regel proprietär und inkompatibel zueinander sind [KKSM02, 45]. Insbesondere bei Standardsoftware besteht darüber hinaus kaum eine andere Möglichkeit, als die systemspezifischen Autorisierungskomponenten zu verwenden.

Bei heterogenen Systemlandschaften erfolgen die Verwaltung und Kontrolle der Berechtigungen in der Praxis aufgrund der proprietären Komponenten weitestgehend systembezogen, so dass eine systemübergreifende Transparenz über die vergebenen Berechtigungen und eine effiziente Administration nur eingeschränkt realisiert werden [KKSM02, 45]. Insbesondere die mangelnde Transparenz steht dabei in Widerspruch zu steigenden regulatorischen Anforderungen [HPJH03, 13; MMJK04, 7; Robi05, 26], wie sie z. B. durch den Sarbanes-Oxley-Act [Cong02] aufgestellt werden. Ziel des Beitrags ist es daher, den
State-of-the-Art von Vorgehensmodellen für die Integration der Autorisierung in der Forschung wie auch in der Unternehmenspraxis darzustellen. Die Analyse beider Bereiche bildet den Ausgangspunkt für die Weiterentwicklung eines Vorgehensmodells für die Definition und Implementierung systemübergreifender Rollen zur Verknüpfung systemspezifischer Berechtigungen. Der Aspekt des Datenschutzes wird dabei nur am Rande betrachtet, da er sich auf den Schutz personenbezogener Daten im Dauerbetrieb bezieht und nicht primär auf die projektartige Integration von Berechtigungen über Systemgrenzen hinweg.

Zunächst werden die Grundlagen der Autorisierung erläutert und aktuelle Ansätze zur Integration der Autorisierung aus der Forschung dargestellt. Danach werden aktuelle Praktiken in Großunternehmen in Form zweier Fallstudien analysiert. Der Schwerpunkt der Analyse liegt auf den jeweiligen Vorgehensmodellen. Da die unter-

\section{Kernpunkte}

Der State-of-the-Art in Forschung und Praxis zur Autorisierung in heterogenen Systemlandschaften wird dargestellt. Folgende Ergebnisse können aus der Analyse abgeleitet werden:

- Die existierenden Forschungsbeiträge enthalten nur wenig detaillierte Vorgehensmodelle zur Integration der rollenbasierten Autorisierung.

- Die Praxis hält konkretere Ansätze bereit, die als Ausgangspunkt für verbesserte Vorgehensmodelle dienen können.

- Durch eine Kombination von Erkenntnissen aus Theorie und Praxis kann die Grundlage für ein verbessertes Vorgehensmodell geschaffen werden.

Stichworte: Autorisierung, Administration, Berechtigungsmanagement, Integration, Rollen, Zugriffskontrolle 
suchten Forschungsbeiträge ausführliche Objekt- und Metamodelle, aber nur wenig detaillierte und nicht theoretisch verankerte Vorgehensmodelle enthalten, muss für eine umfassende und verbesserte $\mathrm{Me}$ thode zur Integration der Autorisierung auf die Erkenntnisse aus Theorie und Praxis in Kombination zurückgegriffen werden. Dies erfolgt im abschließenden Abschnitt in Form einer Konsolidierung der Analysen.

\section{Integration der Autorisierung}

Im Folgenden werden zuerst grundlegende Konzepte der Autorisierung wie die rollenbasierte Zugriffskontrolle vorgestellt. Darauf aufbauend wird schließlich der Einsatz von systemübergreifenden Rollen als Mittel zur Integration der Autorisierung diskutiert.

\subsection{Grundlagen der Autorisierung}

Autorisierung oder synonym Zugriffskontrolle [Pern95, 166] bezeichnet die Überprüfung und Verwaltung von Zugriffsrechten [RuWo06, 127]. Die Überprüfung von Zugriffsrechten ist definiert als der Prozess der Vermittlung von Anfragen an Ressourcen und Daten eines Systems und die Entscheidung, ob die Anfrage zugelassen oder zurückgewiesen wird [SaCa02, 137]. Die Verwaltung von Zugriffsrechten umfasst das Erteilen, Entziehen und Pflegen von Zugriffsrechten [RuWo06, 127]. Im Laufe der Zeit wurden unterschiedliche Autorisierungsansätze entwickelt. Diese Ansätze können in drei Klassen eingeteilt werden [Fisc01, 79 f.; SaCa02, 139]:

- Benutzerbestimmte Zugriffskontrolle: Die benutzerbestimmte Zugriffskontrolle basiert auf Zugriffsregeln, die direkt angeben, wer welche Aktionen mit welchen Ressourcen durchführen kann [SaCa02, 139]. Im Englischen wird die benutzerbestimmte Zugriffskontrolle als „Discretionary Access Control“ („Beliebige Zugriffskontrolle“ oder „dem eigenen Ermessen überlassene Zugriffskontrolle") bezeichnet, da der Nutzer die Möglichkeit hat, seine Rechte auch an andere Nutzer weiterzugeben. Die Zugriffsmatrix ist das elementare Konzept für die Beschreibung von benutzerbestimmten Zugriffskontrollkonzepten [SaCa02, 140].
- Systembestimmte Zugriffskontrolle: Die systembestimmte Zugriffskontrolle arbeitet auf der Basis von Sicherheitsmarken [Pern95, 170; SaCa02, 148]. Diese beschreiben zum einen die Vertrauenswürdigkeit des zugreifenden Subjekts. Zum anderen bestimmen sie die Sensitivität einer Ressource. Die Verwaltung und der Abgleich der Sicherheitsmarken von zugreifenden Subjekten und Ressourcen werden durch eine zentrale Instanz geregelt [Fisc01, 80; Pern95, 170;

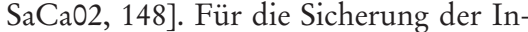
tegrität und der Vertraulichkeit existieren jeweils spezielle Konzepte $[\mathrm{SaCaO2}$, 148]. Im Englischen wird die systembestimmte Zugriffskontrolle in Abgrenzung zur „Discretionary Access Control“ als "Mandatory Access Control“ („Verbindliche Zugriffskontrolle“) bezeichnet.

- Rollenbasierte Zugriffskontrolle: Bei der rollenbasierten Zugriffskontrolle werden die Rechte nicht direkt an die zugreifenden Subjekte vergeben, sondern $\mathrm{zu}$ Rollen zusammengefasst [ $\mathrm{SaCaO2}$, 180]. Die zugreifenden Subjekte werden erst in einem zweiten Schritt den Rollen zugeordnet. Rollen stellen somit die $\mathrm{Zu}-$ sammenfassung von Rechten dar, die zur Erfüllung von mit ihnen verbundenen Aufgaben notwendig sind [LaGe94, 66]. Die Hauptmotivation hinter der rollenbasierten Zugriffskontrolle liegt in der Tatsache, dass bei der Ausführung von Geschäftstätigkeiten nicht die Identität der einzelnen Person, sondern die organisatorischen Verantwortlichkeiten der Person im Vordergrund stehen $[\mathrm{SaCa} 2,180]$.

Als dominierendes Verfahren der $\mathrm{Zu}$ griffskontrolle etablierte sich in den 90er Jahren die rollenbasierte Zugriffskontrolle [HeSc04, 290]. Obwohl für die rollenbasierte Autorisierung unterschiedlichste Ansätze vorgestellt und implementiert wurden, sind ihnen allen doch grundlegende Konzepte gemein [SaCa02, 181]. Maßgeblich vorangetrieben wird die rollenbasierte Zugriffskontrolle vom National Institute of Standards and Technology (NIST) [HeSc04, 290], das den „NIST Standard for RoleBased Access Control (RBAC)“ [FSGK01] zur rollenbasierten Zugriffskontrolle herausgegeben hat. Der Standard definiert Elemente, die typischerweise in rollenbasierten Systemen implementiert sind [FSGK01, 227]. Auf der Basis des RBAC-Standards wurde der ERBAC-Ansatz [KKSM02] entwickelt, der die Integration der Autorisierung thematisiert. Dieser Ansatz ist Gegenstand der folgenden Ausführungen.

\subsection{Integration der Autorisierung auf Basis systemübergreifender Rollen}

Ein/e typische/r Anwender/in benötigt Zugang zu zahlreichen, unterschiedlichen Systemen, die auf diversen Systemplattformen betrieben werden [KKSM02, 45]. Bestehende Berechtigungskonzepte sind in der Praxis jedoch weitestgehend systemspezifisch: Die informationstechnisch abgebildeten Regelungen, die festlegen, welche/r Benutzer/in auf welche Methoden und/oder Datenobjekte zugreift, werden in der Regel unkoordiniert im Kontext einzelner Systeme in den entsprechenden systemspezifischen Komponenten definiert und gepflegt [KKSM02, 45].

Um die systemspezifische, unkoordinierte Rechtevergabe zu überwinden und eine integrierte, effektive und effiziente Administration zu gewährleisten, wurden Konzepte für die systemübergreifende $\mathrm{Zu}$ griffskontrolle entwickelt. Das „Enterprise RBAC Model (ERBAC)" [KKSM02] und seine Erweiterungen [KuSSO3] sind im Kontext dieses Beitrages besonders hervorzuheben, da sie auf der Basis des RBACStandards entwickelt wurden. In Anlehnung an den RBAC-Standard versucht der ERBAC-Ansatz, die grundlegenden Konzepte der rollenbasierten, systemübergreifenden Zugriffskontrolle darzustellen und $\mathrm{zu}$ systematisieren [KKSM02, 46].

Bild 1 [Wort06, 185] zeigt das Modell der systemübergreifenden Autorisierung, das auf dem ERBAC-Ansatz aufbaut, dieses jedoch insbesondere um die ablauforganisatorischen Elemente „Person“, „Prozessrolle" und „Aufgabe“ erweitert. Ausgehend von den ablauforganisatorischen Elementen wird das Modell im Folgenden vorgestellt.

Damit ein/e menschliche/r Aufgabenträger/in („Person“) [Seuf02, 5] die ihm über Prozessrollen [RoMü97, 100f.] zugeordneten, informationstechnisch unterstützten Aufgaben durchführen kann, muss er/sie über entsprechende Systemberechtigungen („Berechtigungen“) verfügen. Diese systemspezifischen Berechtigungen regeln den Zugriff auf die Methoden und Datenobjekte der existierenden Softwarekomponenten und werden über systemspezifische Benutzerkonten („Benutzerkonten Zielsystem“) den Aufgabenträgern zugeordnet [KKSM02, 45 f.]. Die Zuweisung der Berechtigungen zu Benutzerkonten erfolgt in der Praxis über intermediäre, systemspezifische Konstrukte wie Gruppen oder Rollen, die als Ressourcen bezeichnet werden [KKSM02, 46]. 


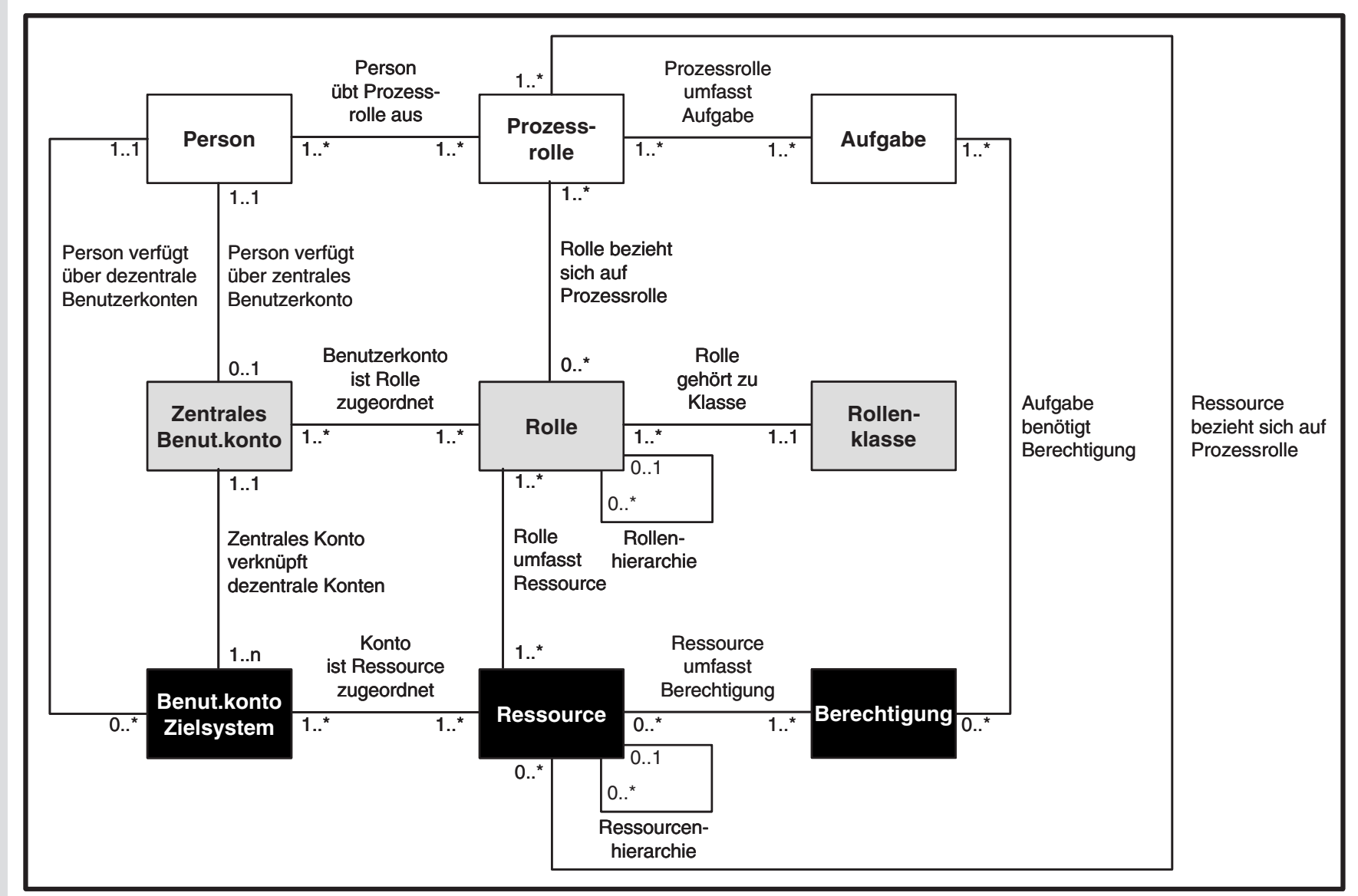

Bild 1 Modell der systemübergreifenden Autorisierung [Wort06, 185]

Die Integration der systemspezifischen Ressourcen erfolgt auf der Basis von Rollen, die die systemspezifischen Ressourcen $\mathrm{zu}$ systemübergreifenden Berechtigungsbündeln zusammenfassen [KKSM02, $45 \mathrm{f}$.]. Im ERBAC-Standard werden die Rollen als Enterprise-Rollen bezeichnet, um ihren systemübergreifenden Charakter hervorzuheben und sie von systemspezifischen Rollen abzugrenzen. Im Mittelpunkt der zu entwickelnden Vorgehensweise steht die Implementierung von systemübergreifenden Rollen. Daher wird der Rollenbegriff im Weiteren im Sinne der Enterprise Rolle gebraucht.

Jede Rolle gehört genau einer Rollenklasse an [RoSW00, 106]. Eine Rollenklasse umfasst eine Menge von Rollen, die aufgrund gemeinsamer Eigenschaften in Abgrenzung $\mathrm{zu}$ anderen Rollen $\mathrm{zu}$ einer Klasse zusammengefasst werden. Rollen werden systemübergreifenden, zentral vorgehaltenen Benutzerkonten zugeordnet [KKSM02, 46], die der Verknüpfung sys- temspezifischer Benutzerkonten dienen und eine systemübergreifende Benutzerrepräsentation darstellen.

Während die in Bild 1 schwarz dargestellten Elemente in dezentralen Autorisierungskomponenten administriert werden, erfolgt die Verwaltung der grau hinterlegten Elemente in einer zentralen, übergreifenden Autorisierungskomponente, die in der Praxis als Enterprise User Administration (EUA) Werkzeug [KKSM02, 45] oder auch Identity Management Werkzeug [KuSS03, 179] bezeichnet wird.

\section{Integration der Autorisierung - Ansätze in der Forschung}

Im Folgenden wird ein Überblick über verschiedene Ansätze gegeben, die sich mit der Einführung und Implementierung systemübergreifender Rollen zur Integration der Autorisierung auseinandersetzen. Eine ausführliche Diskussion dieser Ansätze findet sich in [Wort06, 36-52]. Für die Diskussion wurden Beiträge ausgewählt, die sich im Hinblick auf die Entwicklung eines entsprechenden Vorgehensmodells verwerten lassen und somit (1) einen expliziten Bezug zur systemübergreifenden Autorisierung aufweisen, (2) ein Vorgehensmodell beinhalten oder thematisieren, (3) bezüglich ihres Abstraktionsgrads eine hinreichend konkrete Diskussion erlauben und (4) umsetzungsorientiert sind bzw. bereits in der Praxis eingesetzt wurden. Dementsprechend werden die populären Ansätze OrBAC [ABBB03] und dRBAC [FPPK02] nicht untersucht, da sie keine methodischen Empfehlungen enthalten. Gemäß den genannten Kriterien wird auch der Beitrag zum Role Engineering von Shin et al. [SACJ03] nicht näher untersucht. 
Der Beitrag „Observations on the Role Life-Cycle in the Context of Enterprise Security Management" von Kern et al. präsentiert das ERBAC-Model für die systemübergreifende, unternehmensweite Autorisierung [KKSM02]. Darüber hinaus diskutiert er ein Vorgehensmodell zur Einführung einer unternehmensweiten Autorisierung auf der Basis von systemübergreifenden Rollen. Das Vorgehensmodell ist in vier Phasen unterteilt. In der ersten Phase werden als Aktivitäten die Einführung des EUA-Werkzeugs, die Konsolidierung von Nutzern, Gruppen, Sicherheitsrichtlinien und Zugriffsrechten sowie die Rollenanalyse angeführt. Die Rollenanalyse identifiziert dabei potenzielle Enterprise-Rollen. Im Rahmen der zweiten Phase erfolgt die Aufbereitung der Rollen, so dass diese später in einem Administrationswerkzeug implementiert werden können. In der dritten Phase des Vorgehensmodells erfolgen die Produktivsetzung der rollenbasierten Autorisierung und die Einführung weiterer Administrationswerkzeuge, wie z. B. Passwortsynchronisierungs- oder Administrationsworkflowwerkzeuge. Innerhalb der letzten Phase werden ein Berechtigungsberichtswesen für interne und externe Anspruchsgruppen etabliert sowie die ChangeManagement-Prozesse implementiert. Aufgrund der Kürze des Beitrags werden die wesentlichen Aktivitäten des Vorgehensmodells lediglich kurz charakterisiert.

Der Beitrag „Process-Oriented Approach for Role-Finding to Implement Role-Based Security Administration in a Large Industrial Organization" von Roeckle et al. beschreibt einen Ansatz zur Ableitung von Rollen anhand einer Fallstudie, die die Anwendung der entwickelten Methodik bei Siemens ICN schildert [RoSW00]. Ausgangspunkt der Arbeiten ist dabei die Prozessebene mit den Tätigkeiten, welche die Mitarbeitenden im Rahmen ihrer Arbeit ausführen. Der Fokus der Ausführungen liegt auf der Identifikation systemübergreifender Rollen. Im Mittelpunkt stehen dabei ein Metamodell zur Autorisierung und ein Vorgehensmodell, das den Rollenableitungsprozess beschreibt. Das Vorgehensmodell, das in [Roec99] detaillierter beschrieben wird, legt in einem ersten Schritt die für die Rollenableitung verantwortliche Unternehmenseinheit fest. Die folgenden Aktivitäten beziehen sich auf die eigentliche Definition der Rollen: Nachdem die IT-unterstützten Tätigkeiten identifiziert wurden, erfolgt die Zusammenfassung der Tätigkeiten zu Arbeitsplätzen. Anschließend wird für jeden Arbeitsplatz und jede Tätigkeit eine Rolle bzw. Teilrolle definiert. Berechtigungsbündel, die die Teilrollen mit den entsprechenden Berechtigungen versehen, werden abschließend von Systemadministratoren mit Zugriffsrechten ausgestattet. Die beiden Beiträge beschreiben lediglich die wesentlichen Aktivitäten zur Ableitung der Rollen. Ein detailliertes Vorgehensmodell ist öffentlich nicht zugänglich.

Der Beitrag „Role Mining - Revealing Business Roles for Security Administration using Data Mining Technology“ von Kublmann et al. untersucht die Ableitung von systemübergreifenden Rollen mittels DataMining-Technologien [KuSSO3]. Im Gegensatz $\mathrm{zu}$ anderen methodischen Ansätzen werden die systemübergreifenden Rollen jedoch nicht „top-down“, also ausgehend von der organisatorisch prozessualen Sichtweise, abgeleitet, sondern „bottom-up“. Somit werden die systemspezifischen Rollen und Gruppen mit Hilfe analytischer Verfahren semiautomatisch zu systemübergreifenden Rollen zusammengefasst. Das Vorgehensmodell von Kublmann et al. kann in drei Phasen unterteilt werden. (1) Noch vor der eigentlichen Ableitung der Rollen findet die Zusammenstellung und Aufbereitung der notwendigen Informationen statt. (2) Nachdem erste Erfahrungen mit den gesammelten Daten gemacht wurden, erfolgt die eigentliche $\mathrm{Ab}$ leitung der Rollen. Eine Cluster- und eine Assoziationsanalyse werden verwendet, um die systemübergreifenden Rollen zu definieren. (3) In einer letzten Phase werden die ermittelten Rollen auf ihre Plausibilität hin analysiert und implementiert. Auch die Ausführungen dieses Beitrags sind sehr kurz gehalten, so dass das beschriebene Vorgehen nur auf hohem Abstraktionsniveau nachvollzogen werden kann.

Die diskutierten Vorgehensmodelle für die Einführung und Implementierung systemübergreifender Rollen sind wenig detailliert und weder klar in der Theorie verankert noch transparent aus der Praxis abgeleitet. Daher werden im Folgenden Praxisprojekte in Form von Fallstudien dargestellt, die den Ausgangspunkt für die Ableitung des Vorgehensmodells bilden können.

\section{Integration der Autorisierung - Ansätze in Großunternehmen}

Die beiden im Folgenden analysierten Vorgehensmodelle aus der Praxis wurden auf der Basis von Interviews und Dokumentenanalysen unter Verwendung der Methode „Promet Business Engineering Case Studies" [SeÖs04] als Fallstudien erhoben. Eine ausführliche Beschreibung der Fallstudien findet sich in [Wort06, 53-96]. Die beiden Vorgehensmodelle wurden ausgewählt, da sie Projekte betreffen, in deren Rahmen zahlreiche Berechtigungen unterschiedlicher Systeme auf der Basis systemübergreifender Rollen integriert wurden. Die im Zuge der Fallstudien erarbeiteten Lösungen werden seit mehreren Jahren erfolgreich betrieben und fortlaufend auf weitere Systeme ausgedehnt. Die Lösungen und die entsprechenden Vorgehensweisen haben sich somit in der Praxis bewährt.

\subsection{Fallstudie A: Vorgehens- modell Basler Versicherungen}

Bild 2 zeigt das Projektvorgehen zur Definition und Implementierung der systemübergreifenden Rollen bei den Basler Versicherungen. Dargestellt werden die wesentlichen Projektphasen und ihre Aktivitäten. Die Erarbeitung der Grundlagen erfolgte in parallel durchgeführten Aktivitäten einmalig. Die Definition und Implementierung der Rollen wiederholte sich für jeden Unternehmensbereich.

Vor der eigentlichen Definition und der anschließenden Implementierung der Rollen erfolgte die Erarbeitung wesentlicher Grundlagen durch die Festlegung eines Rollen- und Organisationskonzepts sowie die Inventarisierung der Ressourcen. Im Rahmen der Definition des Rollenkonzeptes (Aktivität 1.1) wurden vier Rollenklassen bzw. -ebenen bestimmt, an denen sich die erstellten Rollen ausrichten. Auf der untersten Ebene befinden sich die „Default-Rollen“, die grundlegende Rechte für alle Mitarbeitenden umfassen. DefaultRollen regeln beispielsweise den Zugriff auf das Intranet oder auf das Mailsystem. Die zweite Ebene umfasst die „AbteilungsRollen“, die elementare Berechtigungen für alle Mitarbeitenden einer Abteilung beinhalten. Die dritte Ebene umfasst die „Standard-Rollen“, die die Berechtigungen umfassen, die mehrere Mitarbeitende einer Abteilung benötigen. Die vierte Ebene beinhaltet die „Spezial-Rollen“, die ausgewählte, besonders kritische Berechtigungen umfassen. Das anschließend (Aktivität 1.2) definierte Organisationskonzept bestimmt die Aufgaben, Kompetenzen und Verantwortlichkeiten bei der Rollendefinition und -pflege. Das Anlegen, Löschen und die Modifikation der Rollen wird durch Rol- 
Erarbeitung der Grundlagen

1.1

Definition des

Rollenkonzeptes

Definition des

Organisationskonzeptes

Inventarisierung der

Berechtigung

Definition der Rollen

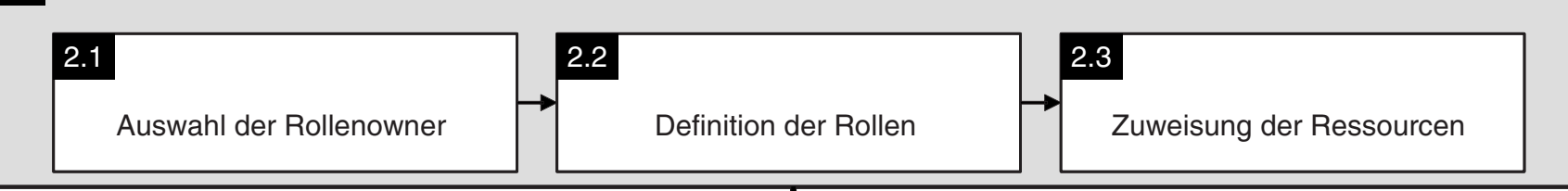

3 Implementierung der Rollen

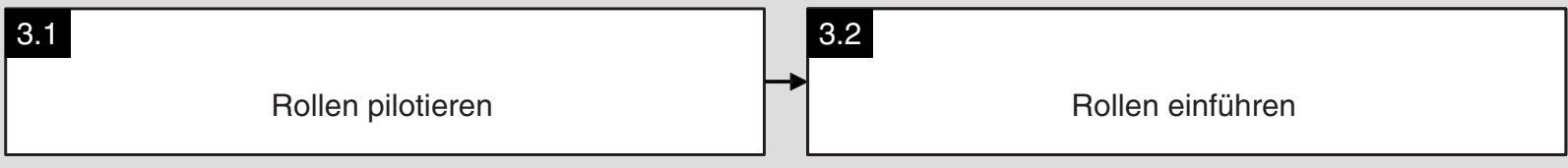

Legende

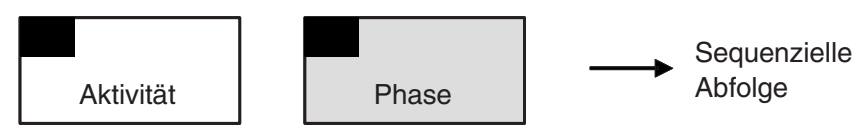

Bild 2 Vorgehensmodell Basler Versicherungen

lenowner verantwortet und durchgeführt. Das Anlegen, Löschen und die Modifikation von Ressourcen obliegt den Ressourcenownern. Um sicherzustellen, dass eine Ressource nur den Rollen zugewiesen wird, die sie auch wirklich benötigen, ist es Aufgabe der Ressourcenowner, Anträge der Rollenowner zur Ressourcennutzung anzunehmen oder abzulehnen. Vor der eigentlichen Definition der Rollen erfolgte die Inventarisierung der Berechtigungen (Aktivität 2.3). Alle Berechtigungen der Systeme und Applikationen wurden in einer hierfür entwickelten Lotus NotesDatenbank aufgenommen, strukturiert und mit einem Kurztext beschrieben. Die Berechtigungen sind dort für alle Mitarbeitenden des Unternehmens einsehbar.

Im Anschluss an die Erarbeitung der Grundlagen erfolgte die Definition der Rollen. Diese Projektphase umfasste die Auswahl der Rollenowner, die eigentliche Definition der Rollen sowie die Zuweisung von Ressourcen. Die Auswahl der Rollenowner (Aktivität 2.1) beinhaltete die Bestimmung der Mitarbeitenden aus dem Fachbereich, die die Rollendefinition für ihren Bereich durchführen und diese im laufenden Betrieb pflegen. Die eigentliche Definition der Rollen (Aktivität 2.2) erfolgte in mehreren Schritten. Zuerst bestimmten die Rollenowner die „Abteilungs-Rollen“, die jedem Mitarbeitenden entsprechend seiner/ihrer Zugehörigkeit zugeordnet werden. Im Anschluss hieran erfolgte die Definition der "Standard-Rollen“, die für ausgewählte Mitarbeitende einer Abteilung relevant sind. Abschließend wurden besonders kritische Berechtigungen durch die Bildung von „SpezialRollen“ berücksichtigt. Nachdem die Rollenowner ihre Rollen definiert hatten, legten sie den Berechtigungsumfang der Rollen fest (Aktivität 2.3).

Im Anschluss an die Definition der Rollen erfolgt die Implementierung der Rollen. Die Implementierung der Rollen wurde in zwei Schritten durchgeführt. Um die reibungslose Einführung der Rollen sicherzustellen, entwickelten die Rollenowner in Zusammenarbeit mit der zentralen Benutzerverwaltung zunächst Rollenpiloten (Aktivität 3.1). Dazu wurde jeweils ein/e repräsentative/r Mitarbeiter/in einer zu im- plementierenden Rolle mit seinem existierenden Berechtigungsprofil detailliert analysiert. Kritisch hinterfragt wurde hierbei insbesondere, ob diese/r Mitarbeiter/in die einzelnen Berechtigungen wirklich benötigt. Auf der Basis dieser Analyse spezifizierte der jeweilige Rollenowner in Zusammenarbeit mit der zentralen Benutzerverwaltung die Rollen mit ihren Ressourcen. Nach der erfolgreichen Pilotierung einer Rolle wurden alle Mitarbeitenden einer Rolle entsprechend angepasst und konfiguriert (Aktivität 3.2). Damit war die Implementierung der Rollen abgeschlossen. Zurzeit existieren ca. 500 Rollen, die über 4.000 Mitarbeiter mit Berechtigungen versorgen.

\subsection{Fallstudie B: Vorgehensmodell GENERALI}

Bild 3 zeigt das Projektvorgehen zur Definition und Implementierung der systemübergreifenden Rollen bei der GENERALI. Dargestellt werden wiederum die wesentlichen Projektphasen und ihre Aktivitäten. 
Vorstudie und Spezifikation erster Lösungsansätze 1.1

Definition „Business-Modell“ der Aufbauorganisation

Definition „Business-Modell“ der Ablauforganisation

2 Definition der grundlegenden Konzepte
2.1

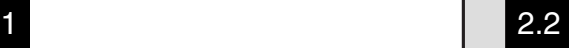
2.2
Definition des
grundlegenden Rollenkonzeptes
$\rightarrow \quad$ Definition der
Rollenstruktur

\section{Festlegung des Ablaufkonzeptes \\ 2.3}

\section{Definition und Implementierung der Rollen}

3.1

Festlegung der Rollen

3.2 mit ihren Ressourcen

\section{$\rightarrow \quad$ Abstimmung der Rollen mit dem Fachbereich}

7

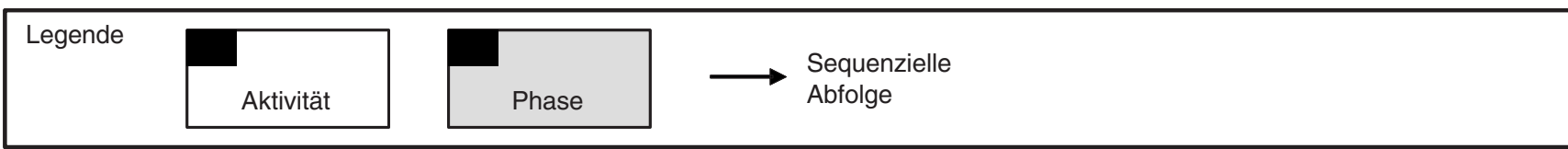

Bild 3 Vorgehensmodell GENERALI

Vor der eigentlichen Definition und Implementierung der Rollen erfolgte die Spezifikation von „Business-Modellen“ im Rahmen der Vorstudie. Die Definition der „Business-Modelle“ der Aufbau- (Aktivität 1.1) und Ablauforganisation (Aktivität 1.2) beantwortete die Frage, wie die Organisation eines Unternehmens mittels technischer Strukturen abgebildet werden kann. Ausgangspunkt des Definitionsprozesses waren betriebswirtschaftliche Definitionen von Aufbau- und Ablauforganisation. Die Erstellung der Modelle berücksichtigte sowohl die primären, dauerhaften Organisationsstrukturen der Unternehmung wie auch die sekundären Organisationseinheiten der Projektorganisation. Zentrale Entität der erarbeiteten Modelle ist die Rolle („Business-Role“), die Aufgabenträger der Aufbauorganisation mit Aufgaben der Ablauforganisation verknüpft. Die definierten Modelle dienten als Diskussions- und Entscheidungsgrundlage zur weiteren Steuerung des Projektes.

Im Zuge der ersten Implementierungen erfolgt die Definition der grundlegenden Konzepte, welche die Spezifikationen des Rollen-, Vererbungs- und Struktur- sowie
Ablaufkonzeptes umfasst. Die Festlegung des Rollenkonzeptes (Aktivität 2.1) beinhaltet die Spezifikation der Rollenkategorien „Business Processes“, „Business Projects" und „Customer Processes". Rollen der Kategorie „Business Processes“ wie z.B. „Individual Life“ umfassen dabei die Berechtigungen, die im Rahmen der internen Organisation zu vergeben sind. Rollen der Kategorie „Business Projects“ umfassen die Berechtigungen, die im Rahmen der Projektorganisation von Projektmitarbeitenden benötigt werden. Die Kategorie „Customer Processes“ enthält die Kundenrollen, die getrennt von der internen Organisation verwaltet werden. Die Rollen der Kategorie „Business Processes“ weisen ein vierstufiges Rollenprofil auf. Rollen vom Typ „Beginner“ enthalten die grundlegenden Berechtigungen eines Bereiches. Rollen vom Typ „Intermediate“ umfassen zusätzliche Berechtigungen. Spezialisten eines Bereiches wird die entsprechende „Expert"-Rolle zugewiesen. Leitenden Mitarbeitenden wird die Rolle „Manager“ zugeordnet. Um eine transparente und einfache Administration $\mathrm{zu}$ gewährleisten, wird im Anschluss an die Definition der
Rollenkategorien ein Vererbungs- und Strukturkonzept für „Business Roles“ definiert (Aktivität 2.2). Im Rahmen der Spezifikation des Ablaufkonzeptes (Aktivität 2.3) erfolgt die Definition der Administrationsprozesse. In Bezug auf die Definition von Rollen muss insbesondere festgelegt werden, welche Organisationseinheiten bzw. welche Mitarbeitenden für die eigentliche Rollendefinition und -implementierung verantwortlich sind.

Das erarbeitete Rollenkonzept sieht pro Unternehmensprozess vier Rollen vor, die es zu implementieren gilt. Die initiale Ausstattung dieser Rollen mit Berechtigungen geschieht durch entsprechende IT-Mitarbeitende mit Unterstützung durch den Fachbereich. Ausgangspunkt der Zuweisung sind zum Definitionszeitpunkt bereits vergebene Berechtigungen. Die Abstimmung der Berechtigungen einer Rolle erfolgt daraufhin mit dem Fachbereich (Aktivität 3.2). Die eigentliche Implementierung der Rollen im Administrationswerkzeug wird wiederum durch IT-Mitarbeitende vorgenommen (Aktivität 3.3). 


\section{Zusammenfassung und Ausblick}

\subsection{Zusammenfassung}

Durch Darstellung des State-of-the-Art in Forschung und Praxis will dieser Beitrag die Grundlage für die Entwicklung eines Vorgehensmodells zur Definition und Implementierung system- und bereichsübergreifender Autorisierungsinfrastrukturen schaffen. Die Analyse zeigt, dass die Ansätze aus der Forschung von den Praktiken erfolgreicher Großunternehmen stark abweichen und generell weniger detailliert sind. Deshalb wird im Folgenden aufgezeigt, wie aus verschiedenen Einzelelementen der Forschungsansätze, insbesondere aber aus den beiden Vorgehens- modellen der Praxis ein konsolidiertes Vorgehensmodell für die Integration der Autorisierung abgeleitet werden kann. Dieses Vorgehensmodell ist als Vorschlag anzusehen, wie die Integration der Autorisierung transparent aus der Praxis und gleichwohl theoriebasiert gestaltet werden könnte.

\subsection{Ausblick: Konsolidierung der} Analysen als Ausgangspunkt für die Entwicklung verbesserter Vorgehensmodelle

Wie bereits angeführt, eignen sich die in Kapitel 3 vorgestellten Forschungsansätze nicht für Empfehlungen für die Integration von systemübergreifender, rollenbasierter Autorisierung. Daher wird zur Entwick- lung des konsolidierten Vorgehensmodells auf die Beispiele der Praxis zurückgegriffen. Aus der Theorie bildet das in Kapitel 2 vorgestellte, aus dem ERBAC-Ansatz weiterentwickelte Objektmodell die Grundlage für das zu konsolidierende Vorgehensmodell. Die Induktion des Vorgehensmodells umfasst zwei Schritte und lehnt sich dabei an die Konstruktion von Referenzmodellen [Broc03; Schü98] an. Eine ausführliche Beschreibung der Ableitung und des Vorgehensmodells findet sich in [Wort06, 180-210].

Zuerst werden die Aktivitäten, die eine sich ähnelnde funktionale Verrichtung umfassen, im induzierten Vorgehensmodell zusammengefasst (vgl. Bild 4). Um die Transparenz der Ableitung zu gewährleisten, werden im abgebildeten Vorgehensmodell für jede abgeleitete Aktivität die

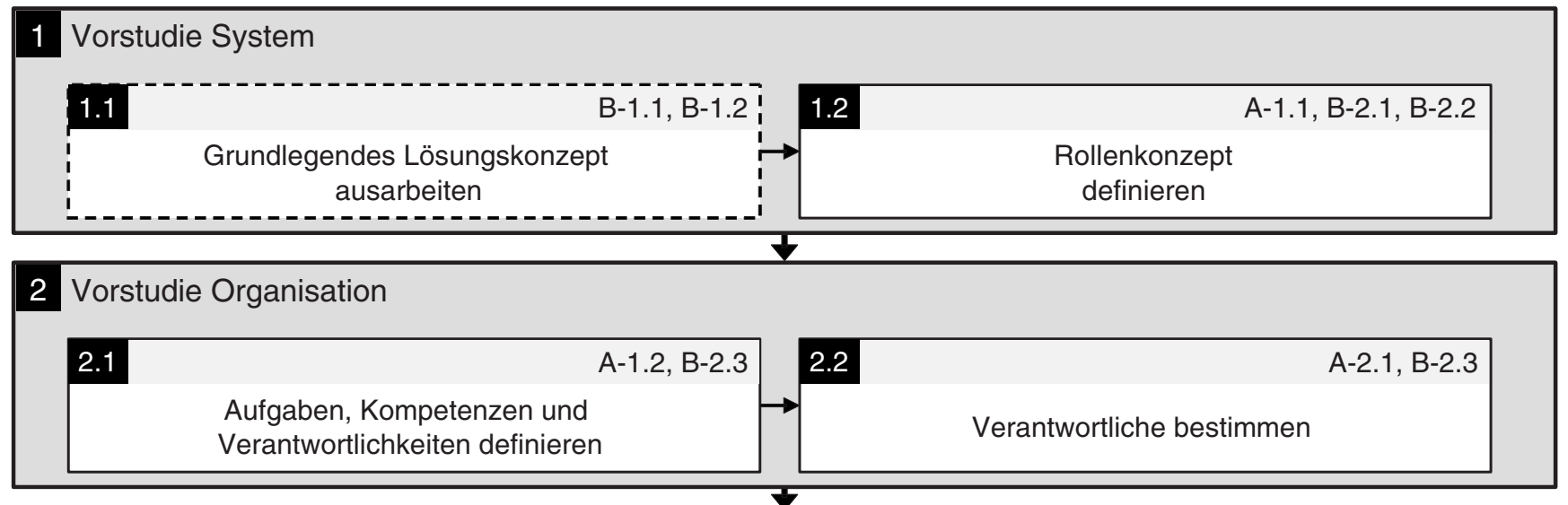

Rollendefinition

3.1 A-1.3

Ressourcen bereinigen

und inventarisieren

A-1.3 $3.2 \quad$ A-2.2, B-3.1

3.3

A-2.3, B-3.2

- - - - - -

Rollenimplementierung

4.1

A-3.1, B-3.2, B-3.3

4.2

A-3.2, B-3.3

Rollen pilotieren

Rollen einführen

\begin{tabular}{|l|l|l|l|l|l}
\hline Legende & Optionale \\
& Aktivität & Phase & Sequenzielle \\
Abfolge
\end{tabular}

Bild 4 Abgeleitetes Vorgehensmodell 
korrespondierenden Aktivitäten der Fallstudien aufgeführt. Eine besondere Rolle bei der Ableitung spielen die Ergebnisse der Aktivitäten. Aktivitäten mit gleichen oder ähnlichen Ergebnissen deuten auf zu konsolidierende Verrichtungseinheiten hin. Die Aktivitäten sind in der Abbildung bereits den induzierten Phasen zugeordnet. Die Ableitung der Phasen erfolgt analog zur Bestimmung der Aktivitäten durch eine Zusammenfassung der Phasen, die ähnliche Verrichtungseinheiten umfassen. Als „optional“ gekennzeichnete Aktivitäten haben ihren Ursprung in lediglich einer der analysierten Fallstudien.

Abschließend erfolgt die Ableitung des eigentlichen Vorgehensmodells. Die einzelnen Phasen mit ihren Aktivitäten werden im Folgenden überblicksartig charakterisiert.

Ziel der Phase „Vorstudie System“ ist es, grundlegende Lösungskonzepte für die Entwicklung und Implementierung der systemübergreifenden Rollen zu definieren. Zum einen werden Möglichkeiten aufgezeigt, wie die Organisation eines Unternehmens mittels technischer Strukturen abgebildet werden kann (Aktivität 1.1). Mit dieser Aktivität geht insbesondere auch die Definition von Begrifflichkeiten zur Schaffung eines einheitlichen Sprachverständnisses einher. Darüber hinaus gilt es, Rollenklassen [RoSW00, 106f.], die in der Praxis auch als Rollenkategorien oder -typen bezeichnet wurden, sowie deren Beziehungen festzulegen (Aktivität 1.2). Die abgeleiteten Rollenklassen strukturieren im weiteren Vorgehen den Rollendefinitions- und -implementierungsprozess. Außerdem bilden sie die Grundlage für die Definition und Zuweisung von Verantwortlichkeiten. Neben den Rollenklassen sind auch die grundlegenden Beziehungen zwischen den Rollen der unterschiedlichen Klassen zu bestimmen. Grundsätzlich erlauben Autorisierungsstandards und -werkzeuge die Vererbung von Berechtigungen [FSGK01, 229 f.], da dies einen reduzierten Administrationsaufwand verspricht. Je nach definierten Rollenklassen ist somit festzulegen, wie viele Vererbungsstufen erwünscht sind und wie die Rollen der unterschiedlichen Klassen zueinander in Beziehung stehen.

In der Phase „Vorstudie Organisation“ werden die Aufgaben, Kompetenzen und Verantwortlichkeiten bei der Definition und Pflege der Berechtigungen zu Administrationsrollen gebündelt (Aktivität 2.1). Hierzu sind zunächst die notwendigen Aufgaben zu identifizieren, die im Rahmen der Definition und Pflege durchzuführen sind. Schließlich müssen die Aufgaben zu
Administrationsrollen gebündelt und mit Kompetenzen und Verantwortlichkeiten versehen werden.

Im Anschluss an die Definition der Administrationsrollen müssen diese mit geeigneten Mitarbeitenden besetzt werden (Aktivität 2.2). Dabei hat ggf. eine Schulung der Mitarbeitenden stattzufinden, um so eine adäquate Wahrnehmung der Administrationsrollen sicherzustellen.

Ziel der Phase „Rollendefinition“ ist es, eine Spezifikation der zu implementierenden Rollen festzulegen. Vor der eigentlichen Definition der Rollen müssen ggf. die Ressourcen, die zu Rollen zu bündeln sind, inventarisiert und bereinigt werden (Aktivität 3.1). Die eigentliche Spezifikation der Rolle beginnt mit der Grobspezifikation der Rollen (Aktivität 3.2). Dabei wird für jede Rolle festgelegt, welche Mitarbeitenden mit der Rolle arbeiten. Darüber hinaus gilt es, die wesentlichen Ressourcen, die eine Rolle umfasst, vorläufig zu bestimmen. Im Rahmen der Fallstudien stellten Organisation und Prozesse den Ausgangspunkt der Rollendefinition dar. Eine Überarbeitung und Anpassung der initialen Rollendefinitionen erfolgt anschließend auf der Basis existierender Berechtigungen. Die ermittelte Grobspezifikation wird sodann verfeinert und abgeschlossen (Aktivität 3.3). Bei der Feinspezifikation der Rollen kommt der Berücksichtigung von Sicherheits- und Datenschutzaspekten eine besondere Bedeutung zu: Im Rahmen der Zuweisung der Ressourcen zu Rollen ist der Schutzbedarf der Ressourcen zu bestimmen, wobei existierende Leitlinien und Vorgaben zu beachten sind. Abschließend ist zu prüfen, ob die Mitarbeiter einer Rolle unter Berücksichtigung des bestimmten Schutzbedarfs auf die der Rolle zugeordneten Ressourcen zugreifen dürfen.

In der Phase „Rollenimplementierung“ werden die definierten Rollen getestet und implementiert. Vor der Implementierung der Rollen empfiehlt es sich, diese zu pilotieren (Aktivität 4.1). Im Rahmen der eigentlichen Rollenimplementierung erfolgt die Einführung der entwickelten Rollen in die Produktion (Aktivität 4.2). Um die Administration weitestgehend $\mathrm{zu}$ automatisieren, bietet sich die Spezifikation von Regeln an, welche die automatisierte Pflege der Berechtigungen unterstützen.

Im Zuge weiterer Forschungsarbeiten sollte auf Basis dieses vorgeschlagenen Vorgehensmodells eine tiefer gehende Absicherung der Ergebnisse auf einer breiteren Fallstudienbasis realisiert werden. Darüber hinaus bietet es sich an, das Vorgehensmodell weiter $\mathrm{zu}$ verfeinern, um detaillierte Handlungsanweisungen für einzelne Aktivitäten zu erhalten. Eine weitere Aufgabe besteht in der Entwicklung eines Referenzprozessmodells, das sich mit den betrieblichen Verwaltungs- und Pflegeprozessen der Administration auseinandersetzt.

\section{- Literatur}

[ABBB03] Abou El Kalam, Anas; El Baida, Rania; Balbiani, Phillipe; Benferhat, Salem; Cuppens, Frédéric; Deswarte, Yves; Miège, Alexandre; Saurel, Claire; Tronessin, Gilles: Organization Based Access Control. In: 4th IEEE International Workshop on Policies for Distributed Systems and Networks (Policy '03). Lake Como, Italy, 2003.

\section{Procedure models for role-based authorization in heterogeneous IS landscapes}

The authors examine how an authorization architecture can be defined which spans various information systems and organizational units. After introducing authorization and architecture fundamentals, related work on authorization, architecture management and role definition is discussed. In particular regarding procedure models for authorization architecture design, these approaches are not very detailed. Moreover they are neither theoretically well-founded nor transparently derived from current industry practices. Therefore two actual industry practices are presented as case studies. By consolidating these practices with findings from current research, a starting point for an improved procedure model for authorization is proposed.

Keywords: Access Control, Architecture, Authorization, Integration, Roles 
[Broc03] vom Brocke, Jan: Referenzmodellierung Gestaltung und Verteilung von Konstruktionsprozessen. Logos, Berlin 2003.

[Cong02] Congress of the United States of America: The Sarbanes-Oxley Act of 2002 http://news.findlaw.com/hdocs/docs/gwbush/ sarbanesoxley072302.pdf, Abruf am 2005-12-06.

[Fisc01] Fischer-Hübner, Simone: IT-Security and Privacy. Springer, Berlin 2001.

[FPPK02] Frendenthal, Eric; Pesin, Tracy; Port Lawrence; Keenan, Edward; Karamcheti, Vijay: dRBAC: Distributed Role-Based Access Control for Dynamic Coalition Environments,. In 22nd International Conference on Distributed Computing Systems (ICDCS). Vienna, Austria 2002.

[FSGK01] Ferraiolo, David: Sandhu, Ravi; Gav rila, Serban; Kubn, Richard D.; Chandramouli Ramaswamy: Proposed NIST Standard for Role-Based Access Control. In: ACM Transactions on Information and System Security 4 (2001) 3, S. 224-274.

[HeSc04] Herwig, Volker; Schlabitz, Lars: Unternehmensweites Berechtigungsmanagement. In Wirtschaftsinformatik 46 (2004) 4, S. 289-294.

[HPJH03] Hartje, Hendrik; Probst, Uwe; Jäck, Klaus; Hessler, Matthias: SAP Berechtigungs wesen - Design und Realisierung von Berechtigungskonzepten für SAP R/3 und SAP Enterprise Portal. Galileo Press, Bonn 2003.

[JoDi94] Jonscher, Dirk; Dittrich, Klaus: Realisierung von Sicherheitsstrategien mit Hilfe flexibler Zugriffskontrollmechanismen. In: Banknecht, Kurt; Dittrich, Klaus (Hrsg.): Sicherheit in Informationssystemen. vdf, Zürich 1994, S. 23-52.

[KKSM02] Kern, Axel; Kublmann, Martin; Schaad Andreas; Moffett, Jonathan D.: Observations on the Role Life-Cycle in the Context of Enterprise Security Management. In: Proceedings of the 7th ACM Symposium on Access Control Model and Technologies. Monterey 2002, S. 43-51.
[KuSS03] Kublmann, Martin; Shohat, Dalia; Schimpf, Gerhard: Role Mining - Revealing Business Roles for Security Administration Using Data Mining Technology. In: Proceedings of the 8th ACM Symposium on Access Control Models and Technologies. Como 2003, S. 179186.

[LaGe94] Lau, B.; Gerhardt, W.: Ein rollenbasiertes unternehmensbezogenes RechteverwaltungsParadigma. In: Bauknecht, Kurt; Teufel, Stephanie (Hrsg.): Sicherheit in Informationssystemen. vdf, Zürich 1994, S. 53-89.

[MMJK04] Menzies, Christof; Martin, Alan; Jourdan, Catherine; Koch, Michael; Strohm, Anja; Heinze, Thomas: Sarbanes-Oxley Act. SchäfferPoeschel, Stuttgart 2004

[Pern95] Pernul, Günther: Information Systems Security - Scope, State-of-the-art and Evaluation of Techniques. In: International Journal of Information Management 15 (1995) 3, S. 239- 255.

[Robi05] Robinson, Teri: Data Security in the Age of Compliance. In: netWorker 9 (2005) 3, S. 2430.

[Roec99] Roeckle, Haio: Rollenbasierter Zugriffsschutz - Automatisierte Bildung der Rollen im Unternehmen auf der Basis eines prozessorientierten Vorgehensmodells. In: IT-Sicherheit 5 (1999) 1, S. 25-34.

[RoMü97] Rosemann, Michael; zur Müblen, Michael: Modellierung der Aufbauorganisation in Workflow-Management-Systemen - Kritische Bestandsaufnahme und Gestaltungsvorschläge. In: Proceedings des EMISA-Fachgruppentreffen 1997. Darmstadt 1997, S. 100-118.

[RoSW00] Roeckle, Haio; Schimpf, Gerhard; Weidinger, Rupert: Process-Oriented Approach for Role-Finding to Implement Role-Based Security Administration in a Large Industrial Organization. In: Proceedings of the fifth ACM workshop on Role-based access control, Berlin 2000, S. $103-110$.
[RuWo06] Rupprecht, Josef; Wortmann, Felix: Zugriffskontrolle in heterogenen Applikationslandschaften. In: Schelp, Joachim; Winter, Robert (Hrsg.): Integrationsmanagement. Springer, Berlin 2006 .

[SaCa02] Samarati, Pierangela; de Capitani di Vimercati, Sabrina: Access Control - Policies, Models and Mechanisms. In: Focardi, Ricardo Gorrieri, Roberto (Hrsg.): Foundations of Security Analysis and Design - Tutorial Lectures. Springer, Berlin 2002, S. 137-196.

[SACJ03] Shin, Dongwan; Ahn, Gail-Joon; Cho, Sangrae; Jin, Seunghun: On Modeling Systemcentric Information for Role Engineering. In Proceedings of the eighth ACM symposium on Access control models and technologies (SACMAT03). ACM Press, Como, Italy 2003 S. 169-178.

[Schü98] Schütte, Reinhard: Grundsätze ordnungsmässiger Referenzmodellierung. Gabler, Wiesbaden 1998.

[SeÖs04] Senger, Enrico; Österle, Hubert: Promet Business Engineering Case Studies (BECS). Arbeitsbericht BE HSG/BECS/1, Institut für Wirtschaftsinformatik, Universität St. Gallen, St. Gallen 2004.

[Seuf02] Seufert, Steffen: Der Entwurf strukturierter rollenbasierter Zugriffskontrollmodelle. In: Informatik - Forschung und Entwicklung 17 (2002) 1, S. 1-11.

[Wint03] Winter, Robert: An Architecture Model for Supporting Application Integration Decisions. In: Proceedings of the 11th European Conference on Information Systems. Neapel 2003.

[Wort06] Wortmann, Felix: Entwicklung einer Methode für die unternehmensweite Autorisierung Dissertation, Universität St. Gallen, St. Gallen 2006 